\title{
Genomic characterization of MDR/XDR-TB in Kazakhstan by a combination of high- throughput methods predominantly shows the ongoing transmission of L2/Beijing 94-32 central Asian/Russian clusters
}

\author{
B. J. Klotoe ${ }^{1}$, S. Kacimi ${ }^{1}$, E. Costa-Conceicão ${ }^{1}$, H. M. Gomes ${ }^{1,2}$, R. B. Barcellos ${ }^{1,3}$, S. Panaiotov ${ }^{1,4}$, D. Haj Slimene ${ }^{1,5}$, \\ N. Sikhayeva ${ }^{6}$, S. Sengstake ${ }^{7}$, A. R. Schuitema ${ }^{7}$, M. Akhalaia $^{8}$, A. Alenova ${ }^{9}$, E. Zholdybayeva ${ }^{6}$, P. Tarlykov ${ }^{6}$, \\ R. Anthony ${ }^{7}$, G. Refrégier ${ }^{1}$ and C. Sola ${ }^{1 *}$ (D)
}

\begin{abstract}
Background: Kazakhstan remains a high-burden TB prevalence country with a concomitent high-burden of multidrug resistant tuberculosis. For this reason, we performed an in depth genetic diversity and population structure characterization of Mycobacterium tuberculosis complex (MTC) genetic diversity in Kazakhstan with both patient and community benefit.

Methods: A convenience sample of 700 MTC DNA cultures extracts from 630 tuberculosis patients recruited from 12 out of 14 regions in Kazakhstan, between 2010 and 2015, was independently studied by high-throughput hybridization-based methods, TB-SPRINT (59-Plex, $n=700$ ), TB-SNPID (50-Plex, $n=543)$. DNA from 391 clinical isolates was successfully typed by two methods. To resolve the population structure of drug-resistant clades in more detail two complementary assays were run on the L2 isolates: an IS6110-NTF insertion site typing assay and a SigE SNP polymorphism assay.
\end{abstract}

Results: Strains belonged to L2/Beijing and L4/Euro-American sublineages; L2/Beijing prevalence totaled almost 80\%. $50 \%$ of all samples were resistant to RIF and to INH., Subtyping showed that: (1) all L2/Beijing were "modern" Beijing and (2) most of these belonged to the previously described 94-32 sublineage (Central Asian/Russian), (3) at least two populations of the Central Asian/Russian sublineages are circulating in Kazakhstan, with different evolutionary dynamics.

Conclusions: For the first time, the global genetic diversity and population structure of M. tuberculosis genotypes circulating in Kazakhstan was obtained and compared to previous local studies. Results suggest a region-specific spread of a very limited number of L2/Beijing clonal complexes in Kazakhstan many strongly associated with an MDR phenotype.

Keywords: Kazakhstan, Tuberculosis, MDR-TB, XDR-TB, Genomics, Public health, Molecular evolution, High-throughput diagnostics methods

\footnotetext{
* Correspondence: christophe.sola@i2bc.paris-saclay.fr

'Institute for Integrative Biology of the Cell (I2BC), CEA, CNRS, Univ. Paris-Sud,

Université Paris-Saclay, 91198 Gif-sur-Yvette cedex, France

Full list of author information is available at the end of the article
}

(c) The Author(s). 2019 Open Access This article is distributed under the terms of the Creative Commons Attribution 4.0 International License (http://creativecommons.org/licenses/by/4.0/), which permits unrestricted use, distribution, and reproduction in any medium, provided you give appropriate credit to the original author(s) and the source, provide a link to the Creative Commons license, and indicate if changes were made. The Creative Commons Public Domain Dedication waiver (http://creativecommons.org/publicdomain/zero/1.0/) applies to the data made available in this article, unless otherwise stated. 


\section{Background}

Kazakhstan is a Former Soviet Union country in Northern Central Asia with 18.3 million inhabitants. In 2016 it was one of the 30 countries worldwide with the highest burden of multidrug-resistant tuberculosis (MDR-TB) (www.stat.gov.kz and [1]). Apart from resistance to Rifampin (RIF) and Isoniazid (INH) that defines MDR, resistance to streptomycin (SRM), fluoroquinolones (FLQ) and other second-line injectable drugs (SLID) is common in MTC isolates from Russia and in Former Soviet Union (FSU) republics $[1,2]$. Such a phenomenon creates a threat to the effective global control of TB infection. According to a recent World Health Organization (WHO) report, extensively drug-resistant TB (XDR-TB) had by the end of 2016 been reported by 123 countries [1], i.e. in 6 countries more compared to the situation one year earlier [3].

Kazakhstan has established an efficient surveillance system to monitor drug resistance in the past three years, with initially 18 tuberculosis dispensaries (14 regional, 2 urban and 2 zonal), recently increased to 27 $[1,4]$. Phenotypic Drug susceptibility Testing (DST) is performed by 22 laboratories and 12 laboratories are reported to be able to run Line Probe Assays (LIPAs, Hain Diagnostics, Germany). Among the 30 high MDR-TB burden countries, 14 had MDR/RR-TB cohorts in 2014 with more than 1000 cases [1]. Among these, Kazakhstan, (together with Myanmar and Viet Nam) reported treatment success of more than $75 \%$. In this context, high-throughput predictive genotyping of drug susceptibility testing could complement and be a faster alternative to phenotypic DST or LIPAs, with high reliability if done in a limited number of certified reference laboratories. As such it could help monitoring as well as targeted DST testing for the bacteriologically confirmed cases (9597 in 2014) [5].

TB-SPRINT and TB-SNPID are Nucleic-Acid PCRbased Amplication Tests (NAAT) that provide simultaneous MTC genotyping subspecies identification and first-line (TB-SPRINT) and first and second-line (TB-SNPID) predictive drug-resistance genotyping (see Table 1 for marker description) [6-11]. These assays use the versatility provided by microsphere hybridization systems that are analyzed either by flow cytometry or by fluorescence imaging using highthroughput analytical devices [12, 13]. TB-SNPID is a multiplex ligation-dependent probe amplification (MLPA) assay whereas TB-SPRINT and TB-EFI rely on direct hybridization and on a dual-oligonucleotide priming (DPO) principle [14, 15].

The main objective of this study was to provide an in depth characterization, at the most reasonable cost for public health, of the genetic diversity and population structure of MTC in Kazakhstan, with
Table 1 Drug-resistance markers analyzed in the two highthroughput microbead-based assays

\begin{tabular}{lll}
\hline DR Markers assesed & TB-SPRINT & TB-SNPID \\
\hline rpoB_176_mut & & $\times$ \\
rpoB_516_GAC_wt & $\times$ & \\
rpoB_516_GTC_mut1 & $\times$ & $\times$ \\
rpoB_516_TAC_mut2 & $\times$ & \\
rpoB_522 & & $\times$ \\
rpoB_526_CAC_wt & $\times$ & \\
rpoB_526_TAC_mut1 & $\times$ & $\times$ \\
rpoB_526_GAC_mut2 & $\times$ & $\times$ \\
rpoB_531_TCG_wt & $\times$ & $\times$ \\
rpoB_531_TTG_mut1 & $\times$ & \\
rpoB_531_TGG_mut2 & $\times$ & \\
katG_315_AGC_wt & $\times$ & \\
katG_315_ACC_mut1 & $\times$ & $\times$ \\
katG_315_AAC_mut2 & $\times$ & \\
inhA_-15_C_wt & $\times$ & \\
inhA_-15_T_mut1 & $\times$ & \\
inhA_-16_A_wt & & \\
inhA_-16_G_mut1 & & \\
inhA_-16_G_wt & & \\
inhA_-8_T_wt & & \\
inhA_-8_A_mut1 & $\times$ & \\
gyrA_90- & & \\
91_GCGTCG_wt & & \\
gyrA 94_wt_GAC & & \\
rrs 1401_wt_A & & \\
rrs1402_wt_C & & \\
eis_(-10A)_CACAA & & \\
eis_(-14T)_TACAG & & \\
rpsl-43 & & \\
rpsl-88 & & \\
rplC-460 & & \\
Reference & & \\
\hline
\end{tabular}

both a potential patient (personalized treatment) and community (molecular epidemiology) benefit. An accessory goal was to check the congruence between the two methods as a surrogate for their quality: if they are congruent, they are likely to be both reliable. If so, they could represent a less sophisticated-less costly high throughput alternate to Next Generation Sequencing (NGS) for surveillance of MDR-XDR-TB in resource-limited countries. Moreover, the understanding of the complex L2 lineage's evolutionary history and diversity was recently improved, and a correspondence between 
numerous authors, diverse genetic markers, and diverse naming has been established. This allows us to use Regions of deletions, SNPs and other markers for L2 epidemic clusters definition (Fig. 1) [6, 16-18]. In Russia, China and FSU republics such as Uzbekistan and Kazakhstan where the prevalence of Beijing is high, methods allowing discrimination within the L2/Beijing are essential to allow MDR-TB evolutionary genomics to be studied $[2,19,20]$.

We report in this paper the genotyping results obtained on isolates collected between July 2010 -November 2015, i.e. a set of 700 MTC DNAs representative of 630 patients (70 duplicates DNA) that were provided by the National Center for Biotechnology of the Republic of Kazakhstan in Astana, working in collaboration with the National Center for Tuberculosis Problems in Almaty. We compare our results to those obtained previously and assess the dynamics of L2/L4 in Kazakhstan.

\section{Methods \\ Patients, demography}

Isolates from a total of 630 patients living in Kazakhstan (435 men, 195 female) were included in the study. Patient data were only available for patients recruited in the Kazakh National Tuberculosis Reference Laboratory. New cases of tuberculosis accounted for $35.6 \%$ of the cases $(224$ of 630$)$ and $64.4 \%(n=406)$ were previously treated patients. Among 630 patients with pulmonary tuberculosis $69 \%(n=435)$ were men and $23 \%(n=145)$ were women, $50(8 \%)$ were unknown. The age of TB patients varied from 16 to 80 years, with an average age of 37 years.

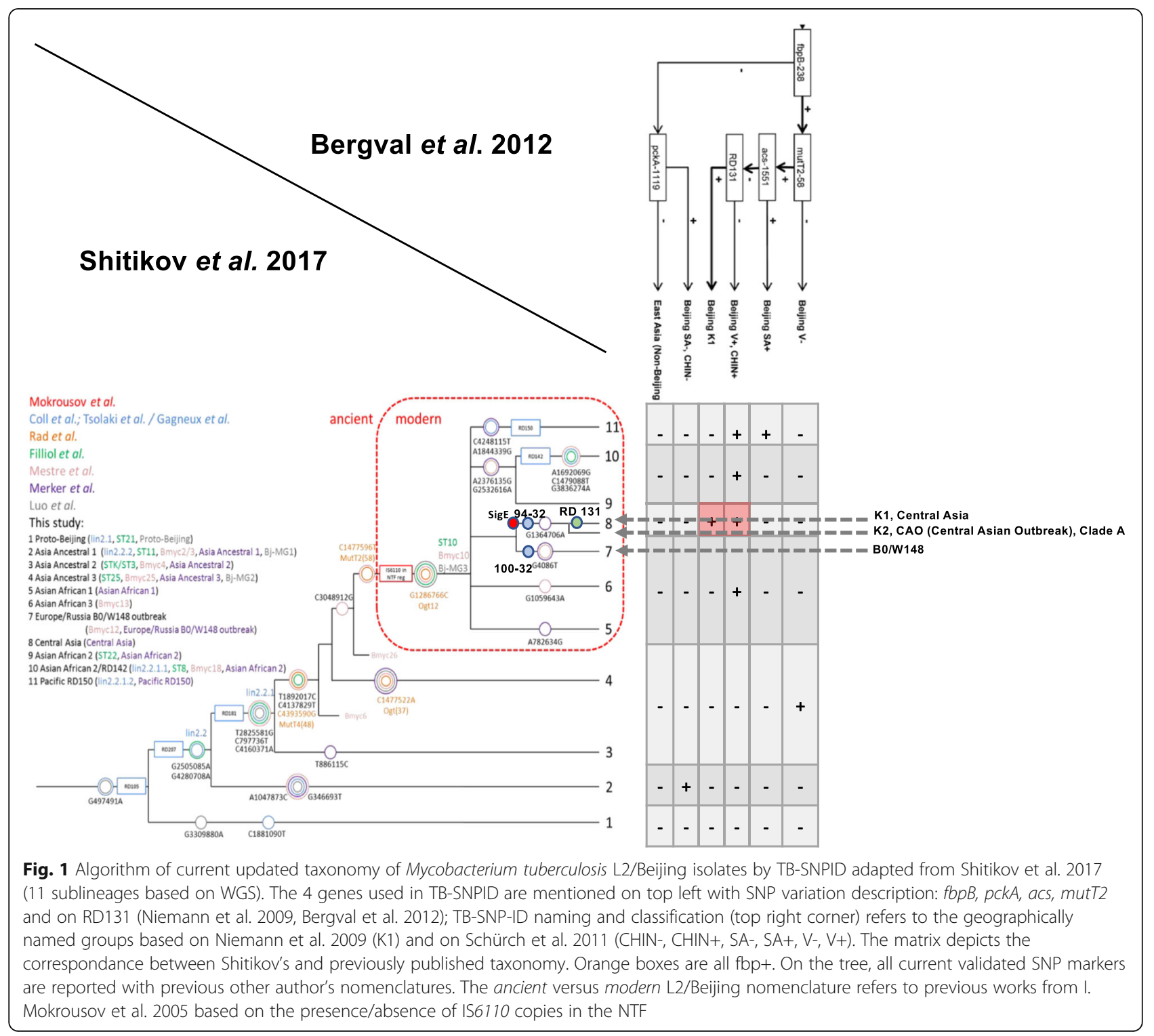




\section{Ethics}

Public health action taken as a result of notification and surveillance is one of the Public Health National Centre for Tuberculosis of Kazakhstan key roles as stated by the Ministry of Health Act and subsequent Government directives which provide a mandate and legislative basis to undertake necessary follow-up. Part of this follow-up is identification of epidemiological and molecular links between cases, helped by the National Center for Biotechnology that provides expertise and links to the Ministry of Research. This study is part of service development carried out under this framework, and as such explicit ethical approval was not mandatory. Moreover patient data are not traceable by any other third part than by the National Centre for Tuberculosis.

\section{Clinical isolates, TB identification, phenotypic DST}

Clinical isolates of MTB were collected from ten regional TB dispensaries (Atyrau, Mangistau, Aktobe, Kostanay, Pavlodar, Semey, Kyzyl-Orda, Zhambyl, Taldykorgan and Almaty) and two prisons run by the State-established committee for the management of the penitentiary system of the Karaganda region and the committee for the management of the penitentiary system of the Akmola region. Isolates were collected from all major regions of Kazakhstan, representing the territory of Kazakhstan. Accordingly, our samples characterize the lineage prevalence of tuberculosis in Kazakhstan as a whole. The high level of sampling from retreated cases however makes it inappropriate to evaluate drug-resistance prevalence in the general population. Clinical isolates of $M$. tuberculosis were also deposited in the Reference Laboratory of the National Center of Phtisiopulmonology for surveillance studies of anti-TB drug resistance in Kazakhstan. After primary isolation on Löwenstein-Jensen (LJ), mycobacteria were subcultured on the same medium in regional laboratories. Species identification as $M$. tuberculosis and drug susceptibility testing were performed at the Reference Laboratory by standard procedures [21]. Rifampicin and isoniazid susceptibility tests were carried out on LJ medium containing $40 \mu \mathrm{g} / \mathrm{L}$ rifampicin, $0.2 \mathrm{mg} / \mathrm{L}$ isoniazid, or $1 \mathrm{mg} / \mathrm{L}$ isoniazid using the absolute concentration method according to the World Health Organization (WHO) recommendations.

\section{DNA extraction methods}

DNA was extracted by thermolysis. Briefly, colonies were pelleted, culture medium was discarded, and colonies resuspended in TE $(10 \mathrm{mM}$ Tris- $\mathrm{HCl}, 1 \mathrm{mM}$ EDTA, pH 7.0). Samples were heated to $95^{\circ} \mathrm{C}$ for 45 $\min$. The suspension was centrifuged at $15,000 \mathrm{~g}$ for 1 min to pellet the cell debris. The supernatant containing the DNA was harvested and transfer into a new tube.
The stocks were stored at $-20^{\circ} \mathrm{C}$ until further use, or diluted at 1:50 into sterile water in a new tube. Seven hundred DNAs were shipped to France and to the Netherlands for further studies in two shipments (June 2015, $n=93$, August 2015, $n=607$ ).

\section{Multiplexed genotyping methods Tb-sprint}

TB-SPRINT typing (59-Plex) is a modular 43-Plex (spoligotyping) plus 16-Plex (first-line drug resistance typing) NAAT developed for Luminex $200^{\circ}$, MagPix ${ }^{\circ}$ and FlexMap3D ${ }^{\circ}$ systems (Luminex Corp, Austin, TX) [22]. In this study, two consecutive experiments, firstly spoligotyping, secondly first-line drug-resistance typing were run. Readings were performed on a Luminex $200^{\circ}$ analyzer using $\mathrm{xMAP}^{\oplus}$ coupled microspheres (Beamedex ${ }^{\circ}$ SAS, Orsay, France). Second-line drug resistance typing was also tested by the new TB-EFI, a 18-Plex method [23]. Full results are shown in (Additional file 1: Tables S1 and S3 677 spoligotyping results with lineage and sublineage and prevalence percentage table).

\section{Tb-SNPID}

TB-SNPID was done as previously described [6] using semi-automated data analysis based on a threshold value as published in [8]. Readings were performed on a MagPix $^{\ominus}$ using the MagPlex xTAG $^{\circ}$ microspheres and reagents provided by MRC Holland (Amsterdam, The Netherlands). DNA samples were first diluted 1:3 in TE containing RNase A (Roche Biochemicals, Pensberg, Germany). Each sample was run in a single well; no samples were repeated. A total of 543 samples were run, however 73 did not pass through the quality check. Hence the final results analysis was performed on 470 samples. Full results are available in (Additional file 1: Table S2).

\section{NTF-analysis insertion typing of IS6110 insertion in L2 isolates}

A PCR-based agarose gel method was used to analyze the presence/absence of $0-2$ copies of IS6110 in the NTF locus of the L2/Beijing isolates DNAs to discriminate between modern and ancient L2/Beijing [24]. Moreover, a microsphere-based method of this original protocol was also developed and confirmed the gelbased results (results not shown, method to be described elsewhere).

\section{SigE polymorphism analysis}

A PCR-RFLP analysis specifically targeting the sigE polymorphism that characterize a mutation specific of the 94-32 L2/Beijing cluster (Central Asian/Russian, including the $\mathrm{K} 1$ strain from Uzbekistan) was also 
implemented $[18,25,26]$. The targeted mutation, when present, creates a double band of 38 and 37 bp by creation of a new AluI site in codon 38 through a CTG - > CTA mutation.

\section{Taxonomic assignations (nomenclature) of molecular results; analysis of 94-32 cluster prevalence}

We applied the latest whole-genome sequencing-based (WGS) taxonomical assignation in 7 lineages (L1-L7) complemented by sublineages (i.e. L4.3 for LAM) when a correspondence between spoligotyping-based naming and NGS-based naming was found [27-30]. VNTR clones are designated according to $[18,25,26]$ and L2/ Beijing sublineages designation was done according to [17]. Of note, in this regard, TB-SNPID performs better than spoligotyping by sub-classifying L2/Beijing isolates in six subgroups based on SNPs and regions of deletions (RD105, mutT2-58, fbpB-238, pckA-1119, acs-1551, RD131). Conversely spoligotyping allows a finer classification of L4 isolates: at least ten accepted sublineages whereas TB-SNPID assigns only four L4 sublineages (L4.1.1/X, L4.3/LAM, L4.1.2/Haarlem, L4/ Euro-American others). Interestingly, TB-SNPID also correctly identifies the previously spoligotypingmisclassified $\mathrm{T}$ signatures, now designated as "LAMRUS” [31, 32].

\section{GIS systems and maps building}

QGIS v3.0 (www.qgis.org, Girona version) was installed on Mas OSX (Apple, Cupertino, CA); Free Kazakhstan shapefiles were downloaded from http://www.diva-gis. org (www.gadm.org, version 2.5, July 2015). Available published results as well as our results were recorded into the required csv file format [33, 34]. Geographical coordinates of main cities in Kazakhstan were downloaded by Google search and and Latitude and Longitude were added to the csv files. Apple pies charts were built following QGIS user's manual.

\section{Statistics}

Chi-squared test or Fisher's exact test were used for all statistical results presented in the study; they were either run using Excel $^{\circ}$ formulas or using an on-line available calculator (https://biostatgv.sentiweb.fr).

\section{Results}

Lineage and intra-lineage MTC genomics characterization using TB-SPRINT and TB-SNPID

TB-SPRINT and TB-SNPID were respectively performed in France and in the Netherlands; each location was blinded to the results generated in the other location. Definitive results from both centers were then compared and cross-checked. One hundred percent identification concordance was obtained on the first studied set of 93 samples (cf. Additional file 1: Table S4, columns E and Q) and $99.3 \%$ of concordant subspecies identification was found at the end of the study with only two unexplained discrepancies. Intra-species typability was better by spoligotyping $96 \%$ typability (23/700 failed) than by TB-SNPID 86.6 typability (73/543 failed). The L2/Beijing represented $79 \%(536 / 677=79.1 \%$ by TB-SPRINT; SIT1 $n=529, \operatorname{SIT} 190 n=5, \operatorname{SIT} 260 n=1, \operatorname{SIT} 1674, n=1$; and $374 / 470=79,5 \%$ using TB-SNPID on an partly independent sample set). The total number of isolates identified as non-L2 by the two methods was around $22 \%$ (96/ $470=20.4 \%$ by TB-SNPID and $24.2 \%=164 / 677$ by TBSPRINT).

The most prevalent L4 sublineage was L4.3/LAM ( $n=$ 51), that includes the so-called LAM-RUS sublineage (previously $\mathrm{T} 1$ or T5-RUS2 in spoligotyping-classification) $[31,32,35]$; the second non-L2 most prevalent lineage was the L4.2.1/URAL genotype [36] $(n=39$, labeled as "Euro-American Other" by TB-SNPID). Five isolates were labeled as "Haarlem" (L4.1.2) by TB-SNPID. By spoligotyping, twelve spoligotypes not reported in the SITVIT database were found, among which 4 were also identified as bona fide L4 isolates by TB-SNPID (others: ND). At least three of these non reported spoligotyping patterns were likely linked to represent mixed infection.

No L2.1 (proto-Beijing) nor L2.2.2 (Asia Ancestral 1) were found based on $f b p B-238$ results in TB-SNPID. As expected, no L2.2.1.1 (Pacific RD150) was found in Kazakhstan based on acs-1551 SNPs. All L2/Beijing identified isolates were further shown to belong to the L2/Modern subgroup (Fig. 1) by the gel-based IS6110NTF method, confirmed by an NTF-IS6110 microsphere-based assay (results not shown). L2 was further split by TB-SNPID into: Beijing SA+ $(n=163$ or $34 \%)$, Beijing $\mathrm{V}+/ \mathrm{CHIN}+/ \mathrm{K} 2(\mathrm{n}=16$ or $3.4 \%)$, Beijing (V-) ( $n=73$ or $15.5 \%)$, Beijing K1 $(n=22$ or $4.6 \%)$. The correspondence between this designation and the current accepted L2 sublineage nomenclature is shown in Fig. 1, for instance $\mathrm{K} 1$ isolates belong to the L2 sublineage 8 from Shitikov et al. [17]. Other L2 epidemic clonal complex are found within these two sublineages [19].

\section{Genotypic predictive drug-resistance results}

Simultaneous precise identification and predictive drug-resistance typing is of great clinical and epidemiological interest. Typeability on drug-resistance conferring mutations was $76 \%$ for TB-SPRINT, and $86.3 \%$ by TB-SNPID. Among the set of samples run by the two methods $(n=391)$, we detected 257 MDR by TB-SPRINT and 247 by TB-SNPID and 75 fully susceptible isolates by TB-SPRINT and 67 by TB-SNPID. All other isolates showed various resistance genotypes (see Additional file 1: Tables S1, S2, S5). MDR prevalence of the isolates in this study lies at $60 \%(n=273 / 470$ i.e. $58.1 \%$ by TB-SNP-ID; 
$n=344 / 515$ i.e. $66.8 \%$ by TB-SPRINT); full susceptibility around $20 \%(93 / 240=19.9 \%$ and $100 / 515=19.4 \%$ respectively); monoresistance to INH around 15\% (86/470 and $67 / 515=13 \%)$ and RIF monoresistance around $2 \%(18 / 470$ and 4/515). These figures do however not represent actual resistance prevalence as our sampling was strongly biased in favor of retreated cases (64.4\% of the total isolates).

Based on TB-SPRINT, 62.3\% L2/Beijing isolates (320/421) were MDR whereas only $4.6 \%$ non-L2/ Beijing isolates were (24/94). The statistical significance of this result is very high (odds ratio $=9.19$ $\left.\mathrm{IC}_{95}[5.39,16.13], p<2.210^{-16}\right)$, showing that $\mathrm{L} 2$ lineage and MDR-TB are strongly associated in Kazakhstan. Table 2 gives a detailed distribution of resistance patterns according to Lineage and sublineage: Beijing $\mathrm{SA}+$ and $\mathrm{V}+/ \mathrm{CHIN}+/ \mathrm{K} 2$ were more likely to be MDR than Beijing $\mathrm{K} 1$ and Beijing $-/-/-/-(p$-value = $1.38 .10^{-7}$, Fisher's exact Test, Additional file 1: Table S8). L4.3 showed a non-significant trend to be more likely MDR as compared to L4.1.2/Haarlem and L4 -EA-other.

RIF resistance associated mutations occurred mostly in rpoB codon 531 (60\%; 273/470 and 320/515). Other RIF resistance associated mutations concerned positions 522 (around 3\%; 13/470 by TB-SNPID; not assessed by TBSPRINT) and 526 (around 2.7\%; 11/470 and 14/515) and 516-GTC (around 1\%; $4 / 515$ by TB-SPRINT, not assessed by TB-SNPID). Some double mutations were detected (14/515 samples analyzed by TB-SPRINT; Additional file 1: Table S1). No mutations were detected in $r p o B$ at codon position 176.

Regarding INH resistance associated mutations, the $k a t G 315 \mathrm{~S}>\mathrm{T}$ mutation was found in more than $70 \%$ of the isolates (348/470 and 385/515), and inh - 15 mutation in around $5 \%$ of the samples (34/470 and 5/515). Some double mutations were detected (15/515 samples analyzed by TB-SPRINT; Additional file 1: Table S1).

EMB resistance as associated mutations in $e m b B 306$ were found in half of the samples (228/470). Second-line resistance associated mutations in relation to SLID and

Table 2 Detailed combined Identification and predictive drugresistance results obtained by TB-SNPID on first-line drugs

\begin{tabular}{lllllll}
\hline & Total & $\begin{array}{l}\text { S monoR- } \\
\text { INH }\end{array}$ & $\begin{array}{l}\text { monoR- } \\
\text { RIF }\end{array}$ & MDR EMB \\
\hline Euro-American Other & 39 & 25 & 9 & 1 & 4 & 2 \\
LAM (L4.3) & 51 & 12 & 10 & 9 & 20 & 1 \\
Haarlem (L4.1.2) & 5 & 3 & 1 & 0 & 1 & 1 \\
L2/Beijing/K1 & 22 & 4 & 6 & 2 & 10 & 7 \\
L2/Beijing/N+/CHIN+/ & 116 & 2 & 15 & 2 & 97 & 92 \\
K2 & 163 & 5 & 35 & 1 & 122 & 115 \\
L2/Beijing/SA+ & 73 & 12 & 10 & 3 & 18 & 10 \\
L2/Beijing/-/-/-/ & 1 & 0 & 0 & 0 & 1 & 0 \\
/-/-/-/ & & & & & & \\
\hline
\end{tabular}

FQ resistance were detected in decreasing order: the eisG-10A mutation (72/470), rrs1401 (34/470), gyrA94 mutations (30/470), gyrA90 mutations (22/470), eisG-14 $T$ (7/470; Additional file 1: Table S2).

\section{In depth georeferenced characterization of L2/Beijing and first attempt to characterize the evolutionary dynamics of L2/L4 clones circulating in Kazakhstan}

To get a deeper insight on L2 clones circulating in Kazakhstan, we further undertook complementary genomic analysis on a total of $n=356$ DNAs (seven L4 as controls, three "failed" by TB-SNPID, and 346 L2/ Beijing isolates). We used the recently described SigE SNP characterization method which was shown to be specific of the 94-32 Central Asian/Russian L2/Beijing cluster $[18,25,26]$. We obtained 314 mutants out of 346 L2-isolates characterized (90\%), which demonstrates the presence of a limited number of epidemic clones belonging to the Beijing 94-32 cluster. We show that at least two different L2/Beijing epidemiologically linked clusters were circulating in the prison in Stepnogorsk, with at least respectively 20 cases (94-32 cluster) and 8 cases (L2/Beijing second cluster), whereas in Aktobe, a single drug-sensitive L2 clone was circulating during the studied time-frame. This clonal complex is encompassed in the TB-SNPID L2/Beijing/-/-/-/ label (Additional file 1: Table S7).

We then performed a thorough georeferencing using a geographic information system (GIS), synthesizing TB outbreak information of a 15-year period (2001 to 2015). Results are shown in Fig. 2. In the 2005 study published by $\mathrm{T}$. Kubica et al. on 150 patients recruited in 2001 in 9 provinces of Kazakhstan, only 91 isolates could be georeferenced (Fig. 2a). L4 looks quite prevalent at that time compared to L2/Beijing (Fig. 2, left, top), especially in the south-east of the country (Almaty). In the 2015 study published by Y. Skiba et al. on a total of 152 patients recruited in 2008 in 9 provinces of Kazakhstan, a trend towards an increase of the L2/Beijing prevalence can be noticed, with a still high prevalence of L4 in Almaty (Fig. 2a, central). However in our study, performed on a sample of 632 patients in 13 regions, the prevalence of the blue lineage (L2/Beijing) outnumbers the L4, at least in some regions (Fig. 2a, bottom), including Almaty. A statistical analysis demonstrates that in Almaty, the proportion between L2 and L4 is not homogeneous (Chi2 $=52,5 ; p<0.001 ; \mathrm{DF}=2)$; there is a recent higher prevalence of L2 compared to L4, the same is true in Kostanay (however in that setting, only two time points were available, in 2001 and in 2010-2015). No other statistically significant results were obtained regarding tuberculosis lineage distribution evolution. Of note in the Kyzyl-Orda region where L4 seems to increase between the 2008 and 2010-2015 period, the 


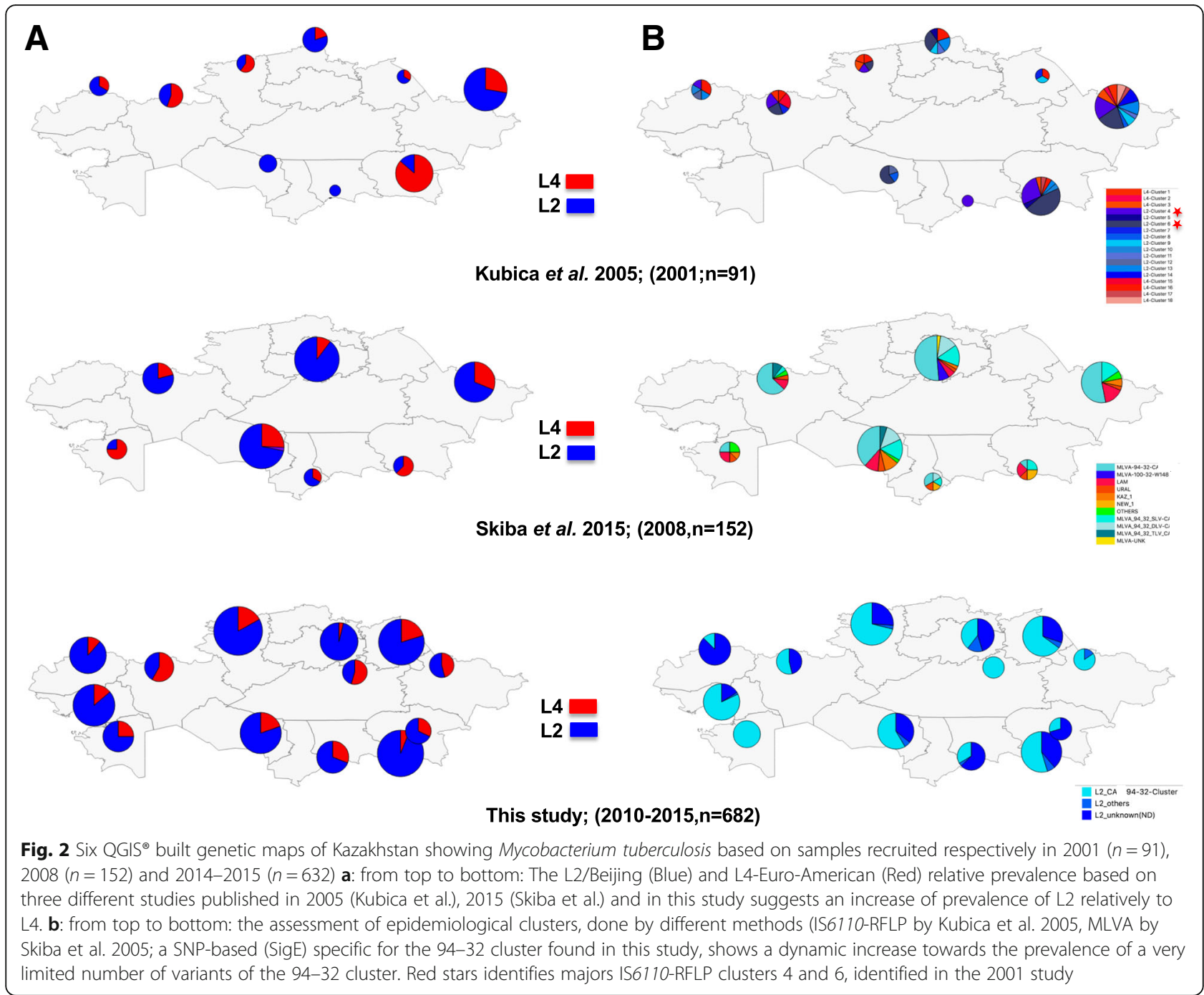

trend was not statistically significant (see Additional file 1 : Table S7).

In Fig. 2b, we retrospectively analyzed the evolution of the prevalence of the L2/Beijing epidemic clones in Kazakhstan, as they had been characterized by different methods: by IS6110-RFLP in the first study, by 24 VNTR typing in the second study, and by combination of various markers (including RD131 and SigE SNP polymorphism) in our study. Figure $2 \mathrm{~b}$ shows that a limited number of clones (likely IS6110-RFLP clusters 4 and 6 in the 2005 article and Beijing 94-32 cluster in the 2015 article) were already circulating in Kazakhstan between 2001 and 2008 (Fig. 2b, top and central). A further comparison of the IS6110-RFLP K1-K2 patterns obtained in a study run in Uzbekistan (isolates obtained during 2001-2004) showed that some IS6110-RFLP clusters found in Kazkakhstan were highly similar if not identical to the IS6110-RFLP patterns described simultaneously in the Uzbekistan study (Additional file 2: Figure S3) [37].
We performed preliminary typing on 8 VNTR on a limited set of L2/Beijing DNA and obtained results that were similar to those obtained by Skiba et al. 2015 confirming the high prevalence of the 94-32 cluster (results not shown). Finally, the last map of Fig. 2b (bottom), provides the SigE polymorphic results, and shows a clear geographic picture of L2/Beijing 94-32 MDR and XDR transmission that suggests an increase of the transmission of the 94-32 L2/Beijing Central Asian/Russian clusters in Kazakhstan.

\section{Discussion}

In this study, we first performed an in depth genomic characterization of MTC isolates circulating in Kazakhstan over an extended sampling time frame (5 years) using a combination of high-throughput NAATs, with the global aim of simultaneously improving MDR-TB surveillance as well as providing the possibility to more accurately personalize TB treatment; complementary genomic 
characterization using IS6110 copy number characterization in the NTF region demonstrates the exclusive presence of Modern L2/Beijing strains in Kazakhstan. Further SigE polymorphism analysis, together with georeferencing, gives a deep dynamic picture of the 2010-2015 TB outbreak in this country; we suggest that the L2/Beijing outbreak in Kazakhstan is essentially linked to the increased spread of 94-32 L2/Beijing clusters, with regional specificities such as in Almaty, Aktobe and to a lesser extent Kostanay, that may deserve further studies using NGS, in order to put these results into a wider phylogeographic context and to compare with the situation in the neighboring Uzbekistan [2] and Kyrgyzstan [38]. A new whole-genome based study that could characterize further the isolates identified on the basis of their SNPs from the Central Asian/Russian and CAO outbreak clones would provide a deeper insight into the genetics of these strains of TB in Kazakhstan [26].

Secondly we demonstrated the use of TB-SNPID, a 50Plex assay that combines species, subspecies and sometimes (for targeted genotypes) highly precise clonal identification simultaneously to drug-resistance gene markers; and we used a new spoligotyping - first-line drug typing direct hybridization assay [6, 8, 22, 39-42]. When independently run on crude DNA extracts these methods proved to be highly congruent, only partially redundant and quite complementary on a set 391 clinical samples that were blindly typed by both methods.

This study is the first of its kind to characterize as precisely, at a national and population level, a large set of TB isolates by genomic non-NGS methods.

\section{Advantages, inconvenience of and discrepancies between the two NAATs}

In this study, TB-SPRINT and TB-SNPID showed some discrepancies regarding the presence of mutations conferring RIF or INH resistance. Part were intrinsically linked to differences in assay design as shown in Table 1. A thorough discrepancy analysis was undertaken on the first set of 93 isolates (Additional file 1: Table S4). This set of samples showed that only one true and unexplained discordance (red color in Additional file 1: Table S4) was found between the two NAATs. The second discrepancy analysis on all 391 results detected 34 discrepancices between the two NAATs $(91.3 \%$ of congruence). We did not re-run discrepant results because of lack of budget. Full results are available in Additional file 1: Tables S5 and S6.

Phenotypic DST MDR status was almost always correlated with the NAAT results ( $n=9$ discrepancies). For six cases, there was a partial agreement on INH resistance, for three others on RIF resistance. Our assays showed altogether limited discrepancies between phenotypic and genotypic DST, in relation to their simple design compared to the complexity of drug resistance emergence and adaptation mechanisms [43]. Discrepancies observed in this study between phenotypic and genotypic DST may be due to errors in phenotypic DST or in genotypic DST since budget was limited to rerun some samples. Infections with mixed resistance genotypes in the patient population may account for another proportion of these discrepancies [44]. Even if phenotypic DST is often still considered as the reference standard, there are inherent difficulties linked to this method, that require quality assurance procedures to track potential errors [45]. In United Kingdom, the switch from phenotypic to genotypic DST is a new reality [46].

Both TB-SPRINT and TB-SNPID methods have advantages and inconveniences: one advantage of TB-SNPID is the "one tube/one run" concept on the $\mathrm{MagPix}^{\circ}$ whereas TB-SPRINT is more suited to Luminex $200^{\circ}$ or FlexMap 3D ${ }^{\circ}$ users. Compared to WGS, TB-SPRINT and TB-SNPID are targeted assays that detect what they are designed to, i.e. a remaining number of samples will always need to be sequenced in particular to discover new mutation responsible of drug resistance. This provides the possibility to adapt these assays to detect specific genotypes. In contrast, WGS is a universal genotyping approach [43]. We recently reported second-line drug resistance assays in Kazakhstan using the 18-Plex "TB-EFI" that targets mutations in eis, gyrA, rrs, embB [23]. Such an assay can be run for a very limited cost in settings where MDR and preXDR-TB is present. Other assays such as the Deeplex ${ }^{\circ}$ from Genoscreen (Lille, France) are other alternative solution to NGS [47]. Predictive WGS-based bioinformatical pipelines represent an important step forward for TB drug-resistance containment and these methods will progressively spread even if they remain quite expensive (200 US\$ per isolate) [48] and considering that bioinformatical pipelines are in evolution [49]. Reagent costs are 20 Euros/sample for TB-SNPID and 12 euros/sample for TB-SPRINT. A raw economic analysis suggest a doubling cost to produce final results using european standard labor costs; these costs are lower in emerging economies. Hence, our assays are cheaper and easier to implement on large numbers of samples than NGS studies for any national, population-based, drug-resistance evaluation study.

\section{Main circulating sublineages in Kazakhstan and MDR transmission risk}

Using both general markers provided by the two NAATs and more specialized markers (RD131 in TBSNPID, SigE run independently) we identified that main circulating clones belong to the L2-Beijing Central Asian/Russian 94-32 sublineage. Further characterization could be performed using the 24 
specific SNPs identified by the recent in-depth WGSbased analysis of L2/Beijing 94-32 cluster [18].

We also showed that some but not all of these 94-32 clones were associated with an MDR genotype: the K1 clone was in contrast linked to susceptibility [37]. We now have a better picture of drug-resistance mutations, and of L2 and non L2 sublineages circulating in Kazakhstan.

\section{Surveillance of L2/Beijing MDRs}

The L2 taxonomy still needs reconciliation between "ancient" knowledge on IS6110-RFLP, VNTR, RDs, and "modern" WGS acquired results to achieve a unified, deeper, and consensus taxonomical definition of L2 epidemic clones $[17,26]$. In addition to purely phylogenetic markers, other polymorphic markers should be investigated to better understand the epidemic success of various clones. Markers of particular interest are undoubtedly adaptive compensatory mutations such as those acting on rpoA and rpoC genes that lower or eliminate the fitness-cost of the drug resistance mutation [19]. An improved global and local knowledge of drug-use history is also of paramount importance to understand the chronology of drug mutation emergence [2, 43]. Looking for epistasis between SNPs, could also be of added value to understand why the 94-32 cluster has been so successful [50].

\section{TB surveillance in high-burden countries}

Achieving improved global tuberculosis control and patient treatment simultaneously is now feasible thanks to WGS or high-throughput SNPs assays. Technology spreading by training should create favorable conditions for sustained laboratory and bioinformatics capacity building or strengthening, and could further be applied to other infectious threats [51, 52]. Genomic assays allow clinical microbiology to change from targeted to nontargeted investigations [53]. However one important issue is the trend towards closed and expensive diagnostics systems development by industrials, that can if not handled carefully contribute to de-skilling and less democratization in health systems. This can generate inequalities due to unaffordable and unsustainable costs as well as creating new local perceptions in populations concerning global health issues if simultaneously linked to austerity policies $[54,55]$. Given the strongly regionalized phylogeographical structure of the TB outbreaks, a "one-size fits all" strategy for MDR-and XDR-TB control may not be the optimal solution; as demonstrated in Swaziland, the prevalence of rare drug resistance mutations such as the $\operatorname{rpoB} \mathrm{I} 491 \mathrm{~F}$, may result in missed MDR-TB cases and inadequate TB treatment and infection control [56]. Global economic issues also remain with respect to sustainability and long-term routine work for WGS-based assays in emerging economies, even if lot of resources are spent to train bioinformaticians [57]. That is the reason why, in the post-genomic era, other locally developed less sophisticated assays, based on and complementary with NGS results, may become important in resource-limited countries [18, 58-60]. Furthermore, the availability of large amounts of WGS data will allow the precise election of genotypes to be screened. They would in turn help to develop secondgeneration assays that could rapidly be applied to very large number of samples.

\section{Conclusions}

In brief, this study demonstrates that two non-NGS information-rich genomic assays could satisfactorily be run independently and successfully, with minimal costs, to produce information-rich public health datasets for patient and community benefit. Our results also suggest the spread of a limited number of L2/Beijing clusters during the 2010-2015 period, with geographic variations and different drug-susceptible/drug-resistant outbreaks histories.

\section{Additional files}

\begin{abstract}
Additional file 1: Table S1. detailed TB-SPRINT results on 700 MTC DNA samples from Kazakhstan ( $n=677$ spoligotypes including 3 potential "mixed infection", 23 failed). Table S2. detailed TB-SNPID results on 470 MTC DNA samples from Kazakhstan ( $n=543$ analysed, 73 failed). Table S3. Lineage and Sublineage assignation of Spoligotyping results $(n=677)$. Table S4. All isolates $(n=93)$ of "set1" (i.e. chronologically first set) typed by two methods: TB-SPRINT and TB-SNPID. Table S5. Detailed results for $n=391$ samples run both by TB-SPRINT and TB-SNPID.

Table S6. Analysis of discrepant drug-resistance results between TBSPRINT and TB-SNPID on the 391 set of isolates. Table S7. congruence between SigE and RD131 (TB-SNPID) results in Aktobe suggests the presence of a single DST L2/Beijing 94-32 clone. Table S8. Chi2 square statistics on $L 2 / L 4$ prevalence in some regions of Kazakhstan. (XLSX $318 \mathrm{~kb}$ )
\end{abstract}

Additional file 2: Figure S3. Comparison between IS6110-RFLP patterns obtained in Kazakhstan (2001) and Uzbekistan. (2001-2004). (PDF 1710 kb)

\footnotetext{
Abbreviations

AMK: Amikacin; DPO: Dual-Priming Oligonucleotide; DST: Drug-susceptibility testing; EMB: Ethambutol; FLQ: Fluoroquinolones; INH: Isoniazid; KAN: Kanamycin; MDR-TB: Multidrug-resistant tuberculosis; MLPA: Multiple ligation-mediated probe dependent amplification assay; MTC: Mycobacterium tuberculosis complex; NAAT: Nucleic acids amplification-based tests; RIF: Rifampin; TB: Tuberculosis; TB-EFI: high-throughput Luminex-based Ethambutol, Fluoroquinolones, second-line Injectable drugs drug-resistance SNPS assay; TB-RINT: high-throughput Luminex-based, first-line drugresistance SNPS assay (Rifampicin, Isoniazid); TB-SNPID: high-throughput Luminex-based MLPA typing and identification SNP-based assay; TBSPOL: high-throughput Luminex-based spoligotyping assay; TB-SPRINT: highthroughput Luminex-based, combined spoligotyping and first-line drugresistance SNPS assay (Rifampicin, Isoniazid); SLID: Second-line injectable drugs; WGS: Whole-Genome Sequencing; XDR-TB: extremely-drug-resistant tuberculosis
} 


\section{Acknowledgements}

We are grateful to the MoH of Kazakhstan, to Dr. Y. M. Ramanculov, Kazakhstan NCB head, for allowing this project to be run, and to Beamedex ${ }^{\oplus}$ (P. Cutolo, M. K. Gomgnimbou, C. Ripoll) and Luminex BV staff in the Netherlands (J. van Gils, E. Benning) for technical support. Hamilton Corporation (Hamilton France, Les Ulis, L. Loridon and J. Vicenzi) is also acknowledged for technical support. Three reviewers helped to improve the paper and are warmly acknowledged.

\section{Authors' contributions}

CS, AA, EZ and PT participated to conception and grant application to the $\mathrm{MoH}$ of Kazakhstan; MA, EZ, RA, SS and CS participated to the study design. SK, ECC, HMG, SP, RCB, NS, BK, ran and analyzed TB-SPRINT assays in Paris. AS, SS, ran and interpreted TB-SNPID assays in the Netherlands. BK developed and ran the IS6110-NTF assays, trained NS and EZ in Kazakhstan and in Orsay. AA provided access to MDR-TB samples with EZ, NS and PT. DHS performed the SNPs-RFLP assay to analyze the 94-32 cluster prevalence. GR ran statistical analysis. GR, CS, SS, RA, EZ, AA coordinated the study respectively in France, Netherlands and Kazakhstan. CS and GR coordinated samples logistics reception and shipment, analyzed results. CA, GR built QGIS maps. $B K, G R, E Z, P T, R A, A A, C S$ wrote the manuscript. CS performed publishing process helped by GR, EZ, PT and RA. All authors read and accepted the final version of the manuscript.

\section{Funding}

This work was supported by the Ministry of Research in Kazakhstan through the project: "High-throughput and cost-effective techniques for control and surveillance of infectious diseases in Kazakhstan: the multi-drug and extremely-drug resistance tuberculosis problem" (Priority area: «Life Science» «Development of biomedicine and genetic engineering») Time frame of the project: January 2015 - December 2017, three years and Grant $n^{\circ}$ AP05130238. BK was a PhD student from Beamedex ${ }^{\circledR}$ supported by the French Agence Nationale de la Recherche et Technologie-Contrat Individualisé de Formation par la Recherche en Entreprise (ANRT-CIFRE n²015-1177 and CNRS-Beamedex contract $\left.n^{\circ} 144729\right)$. CS is grateful to the CNRS and Institute for Integrative Cell Biology, UMR9198, for financial support including the purchase and maintenance of the Hamilton StarLet automatized liquid handling system.

\section{Availability of data and materials}

All raw data are released in the Additional file 1: Tables S1-S8. All material will be made available upon request to the corresponding author.

\section{Ethics approval and consent to participate}

Public health action taken as a result of notification and surveillance is one of the Public Health National Centre for Tuberculosis of Kazakhstan key roles as stated by the Ministry of Health Act and subsequent Government directives which provide a mandate and legislative basis to undertake necessary followup. Part of this follow-up is identification of epidemiological and molecular links between cases, helped by the National Center for Biotechnology that provides expertise and links to the Ministry of Research. This study is part of service development carried out under this framework, and as such explicit ethical approval was not mandatory. Moreover, patient data are not traceable by any other third part than by the National Centre for Tuberculosis.

\section{Consent for publication}

All authors approved the final version of this submission and provided consent for publication.

\section{Competing interests}

CS and GR were among the founders and shareholders of Beamedex ${ }^{\oplus}$, at start of the study. They declare not to hold any share in Luminex Corp. (Austin, TX) nor in Luminex B.V. (Hertogenbosch, The Netherlands), that no relative or parents hold any share either in any of these companies, and that they did not receive any salary or financial counterpart from Beamedex ${ }^{\varpi}$ or Luminex Corp. or Luminex B.V. and that they currently do not hold any Beamedex ${ }^{\oplus}$ share.

\section{Author details}

'Institute for Integrative Biology of the Cell (I2BC), CEA, CNRS, Univ. Paris-Sud, Université Paris-Saclay, 91198 Gif-sur-Yvette cedex, France. ${ }^{2}$ Laboratory of
Molecular Biology Applied to Mycobacteria, FIOCRUZ, Rio de Janeiro, Brazil. ${ }^{3}$ Center of Scientific and Technological Development (CDCT), Secretary of Health of Rio Grande do Sul State (SES/RS), Porto Alegre, Brazil. ${ }^{4}$ National Center of Infectious and Parasitic Diseases, Sofia, Bulgaria. ${ }^{5}$ Institut Pasteur de Tunisie, Tunis, Tunisie. ${ }^{6}$ National Centre for Biotechnology, Astana, Kazakhstan. ${ }^{7}$ Royal Tropical Institute (KIT), Amsterdam, The Netherlands.

${ }^{8}$ Foundation for Innovative New Diagnostics (FIND), Geneva, Switzerland.

${ }^{9}$ National Centre for Tuberculosis Problems, Almaty, Kazakhstan.

\section{Received: 18 October 2018 Accepted: 18 June 2019}

\section{Published online: 24 June 2019}

\section{References}

1. WHO: Global Tuberculosis Report 2017. Geneva: WHO; 2017.

2. Merker M, Barbier M, Cox H, Rasigade JP, Feuerriegel S, Kohl TA, Diel R, Borrell S, Gagneux S, Nikolayevskyy V, et al. Compensatory evolution drives multidrug-resistant tuberculosis in Central Asia. eLife. 2018;7.

3. WHO: Global Tuberculosis Report 2016. Geneva: WHO; 2016.

4. Adenov M, Berikova E, Dzhazybekova P. Implementation of anti-tuberculosis measures in the Republic of Kazakhstan. J Phtisiopulmonol. 2018;1 (1):24-6.

5. WHO: World Healh Statistics 2015. Geneva: WHO; 2015. p. 1-164.

6. Bergval I, Sengstake S, Brankova N, Levterova V, Abadia E, Tadumaze N, Bablishvili N, Akhalaia M, Tuin K, Schuitema A, et al. Combined species identification, genotyping, and drug resistance detection of Mycobacterium tuberculosis cultures by MLPA on a bead-based Array. PLoS One. 2012;7(8):e43240.

7. Gomgnimbou MK, Refrégier G, Sola C. High Throughput combin methods for resistanc emergence analysis, diagnostic and Epidemiology: the multidrug resistant tuberculosis paradigm. In: 10th International Meeting on Microbial Epidemiological Markers. Institut Pasteur; 2013.

8. Sengstake S, Bablishvili N, Schuitema A, Bzekalava N, Abadia E, de Beer J, Tadumadze N, Akhalaia M, Tuin K, Tukvadze N, et al. Optimizing multiplex SNP-based data analysis for genotyping of Mycobacterium tuberculosis isolates. BMC Genomics. 2014;15(1):572.

9. Molina-Moya B, Gomgnimbou MK, Lafoz C, Lacoma A, Prat C, Refregier G, Samper S, Dominguez J, Sola C. Molecular characterization of Mycobacterium tuberculosis strains with TB-SPRINT. Am J Trop Med Hyg. 2017;97(3):806-9.

10. Dantas NGT, Suffys PN, Carvalho WDS, Gomes HM, Almeida IN, Figueiredo LJA, Goncalves AD, Gomgnimbou MK, Refregier G, Sola C, et al. Correlation between the BACTEC MGIT 960 culture system with genotype MTBDRplus and TB-SPRINT in multidrug resistant Mycobacterium tuberculosis clinical isolates from Brazil. Memorias do Instituto Oswaldo Cruz. 2017;112(11):769-74.

11. Santos P, Costa ERD, Ramalho DM, Rossetti ML, Barcellos RB, Nunes LS, Esteves LS, Rodenbusch R, Anthony RM, Bergval I, et al. Detection of tuberculosis drug resistance: a comparison by Mycobacterium tuberculosis MLPA assay versus genotype(R)MTBDRplus. Memorias do Instituto Oswaldo Cruz. 2017;112(6):396-403.

12. Dunbar SA. Applications of Luminex XMAP technology for rapid, highthroughput multiplexed nucleic acid detection. Clin Chim Acta. 2006;363(1-2):71-82

13. Ocheretina O, Merveille YM, Mabou MM, Escuyer VE, Dunbar SA, Johnson WD, Pape JW, Fitzgerald DW. Use of Luminex MagPlex(R) magnetic microspheres for high-throughput Spoligotyping of M. tuberculosis isolates in Port-Au-Prince, Haiti. J Clin Microbiol. 2013;51(7):2232-7.

14. Schouten J, McElgunn C, Waaijer R, Zwijnenburg D, Diepvens F, Pals G. Relative quantification of 40 nucleic acid sequences by multiplex ligationdependent probe amplification. Nucl Acid Res. 2002;30(12):e57.

15. Chun JY, Kim KJ, Hwang IT, Kim YJ, Lee DH, Lee IK, Kim JK. Dual priming oligonucleotide system for the multiplex detection of respiratory viruses and SNP genotyping of CYP2C19 gene. Nucleic Acids Res. 2007;35(6):e40.

16. Mokrousov I, Narvskaya O, Vyazovaya A, Otten T, Jiao WW, Gomes LL, Suffys PN, Shen AD, Vishnevsky B. Russian "successful" clone BON148 of Mycobacterium tuberculosis Beijing genotype: a multiplex PCR assay for rapid detection and global screening. J Clin Microbiol. 2012;50(11):3757-9.

17. Shitikov E, Kolchenko S, Mokrousov I, Bespyatykh J, Ischenko D, llina E, Govorun V. Evolutionary pathway analysis and unified classification of east Asian lineage of Mycobacterium tuberculosis. Sci Rep. 2017;7(1):9227.

18. Mokrousov I, Chernyaeva E, Vyazovaya A, Skiba Y, Solovieva N, Valcheva V, Levina K, Malakhova N, Jiao WW, Gomes LL, et al. Rapid assay for detection of the epidemiologically important central Asian/Russian strain of the 
Mycobacterium tuberculosis Beijing genotype. J Clin Microbiol. 2018;56(2):e01551-17.

19. Casali N, Nikolayevskyy V, Balabanova Y, Harris SR, Ignatyeva O, Kontsevaya I, Corander J, Bryant J, Parkhill J, Nejentsev S, et al. Evolution and transmission of drug-resistant tuberculosis in a Russian population. Nat Genet. 2014:46(3):279-86.

20. Liu Q, Ma A, Wei L, Pang Y, Wu B, Luo T, Zhou Y, Zheng HX, Jiang $Q$, Gan M, et al. China's tuberculosis epidemic stems from historical expansion of four strains of Mycobacterium tuberculosis. Nat Ecol Evol. 2018;2(12):1982-92.

21. David H, Levy-Frebault V, Thorel MF. Méthodes de laboratoire pour Mycobactériologie clinique. In Paris: Institut Pasteur. 1989:1-87.

22. Gomgnimbou MK, Hernandez-Neuta I, Panaiotov S, Bachiyska E, Palomino JC, Martin A, Del Portillo P, Refrégier G, Sola C. "TB-SPRINT: TUBERCULOSISSPOLIGO-RIFAMPIN-ISONIAZID TYPING"; an all-in-one assay technique for surveillance and control of multi-drug resistant tuberculosis on Luminex ${ }^{\circledR}$ devices. J Clin Microbiol. 2013;51(11):3527-34.

23. Klotoe BJ, Molina-Moya B, Gomes HM, Gomgnimbou MK, Suzarte LO, Feres Saad MH, Ali S, Dominguez J, Pimkina E, Zholdybayeva E, et al. TB-EFl, a novel 18-Plex microbead-based method for prediction of second-line drugs and ethambutol resistance in Mycobacterium tuberculosis complex. J Microbiol Methods. 2018.

24. Plikaytis BB, Marden JL, Crawford JT, Woodley CL, Butler WR, Shinnick TM. Multiplex PCR assay specific for the multidrug-resistant strain W of Mycobacterium tuberculosis. J Clin Microbiol. 1994;32(6):1542-6.

25. Allix-Beguec C, Harmsen D, Weniger T, Supply P, Niemann S. Evaluation and user-strategy of MIRU-VNTRplus, a multifunctional database for on-line analysis of genotyping data and phylogenetic identification of Mycobacterium tuberculosis complex isolates. J Clin Microbiol. 2008:46(8):2692-9.

26. Merker M, Blin C, Mona S, Duforet-Frebourg N, Lecher S, Willery E, Blum MG, Rusch-Gerdes S, Mokrousov I, Aleksic E, et al. Evolutionary history and global spread of the Mycobacterium tuberculosis Beijing lineage. Nat Genet. 2015;47(3):242-9.

27. Coll F, Preston M, Guerra-Assuncao JA, Hill-Cawthorn G, Harris D, Perdigao J, Viveiros M, Portugal I, Drobniewski F, Gagneux S, et al. PolyTB: A genomic variation map for Mycobacterium tuberculosis. Tuberculosis (Edinb). 2014;94(3):346-54.

28. Stucki D, Brites D, Jeljeli L, Coscolla M, Liu Q, Trauner A, Fenner L, Rutaihwa L, Borrell S, Luo T, et al. Mycobacterium tuberculosis lineage 4 comprises globally distributed and geographically restricted sublineages. Nat Genet. 2016;48(12):1535-43.

29. Brudey K, Driscoll J, Rigouts L, Prodinger WM, Gori A, Al-Hajoj SAM, Allix C, Aristimuno L, Arora J, Baumanis V, et al. Mycobacterium tuberculosis complex genetic diversity : mining the fourth international spoligotyping database (SpolDB4) for classification, population genetics, and epidemiology. BMC Microbiol. 2006;6(6):23.

30. Demay C, Liens B, Burguière T, Hill V, Couvin D, Millet J, Mokrousov I, Sola C, Zozio T, Rastogi N. SITVITWEB - a publicly available international multimarker database for studying Mycobacterium tuberculosis genetic diversity and molecular epidemiology. Infect Genet Evol. 2012;12(4):755-66

31. Mokrousov I. Molecular structure of Mycobacterium tuberculosis population in Russia and its interaction with neighboring countries. Int J Mycobacteriol. 2015;4(Suppl 1):56-7.

32. Mokrousov I. On sunspots, click science and molecular iconography. Tuberculosis (Edinb). 2018:110:91-5.

33. Kubica T, Agzamova R, Wright A, Aziz MA, Rakishev G, Bismilda V, Richter E, Rusch-Gerdes S, Niemann S. The Beijing genotype is a major cause of drug-resistant tuberculosis in Kazakhstan. Int I Tuberc Lung Dis. 2005;9(6):646-53.

34. Skiba Y, Mokrousov I, Ismagulova G, Maltseva E, Yurkevich N, Bismilda V, Chingissova L, Abildaev T, Aitkhozhina N. Molecular snapshot of Mycobacterium tuberculosis population in Kazakhstan: a country-wide study. Tuberculosis (Edinb). 2015;95(5):538-46.

35. Mokrousov I, Vyazovaya A, Iwamoto T, Skiba Y, Pole I, Zhdanova S, Arikawa K, Sinkov V, Umpeleva T, Valcheva V, et al. Latin-American-Mediterranean lineage of Mycobacterium tuberculosis: human traces across pathogen's phylogeography. Mol Phylogenet Evol. 2016;99:133-43.

36. Kovalev SY, Kamaev EY, Kravchenko MA, Kurepina NE, Skorniakov SN Genetic analysis of Mycobacterium tuberculosis strains isolated in Ural region,
Russian Federation, by MIRU-VNTR genotyping. Int J Tuberc Lung Dis. 2005; 9(7):746-52.

37. Niemann S, Koser CU, Gagneux S, Plinke C, Homolka S, Bignell H, Carter RJ, Cheetham RK, Cox A, Gormley NA, et al. Genomic diversity among drug sensitive and multidrug resistant isolates of Mycobacterium tuberculosis with identical DNA fingerprints. PLoS One. 2009;4(10):e7407.

38. Isakova J, Sovkhozova N, Vinnikov D, Goncharova Z, Talaibekova E, Aldasheva N, Aldashev A. Mutations of rpoB, katG, inhA and ahp genes in rifampicin and isoniazid-resistant Mycobacterium tuberculosis in Kyrgyz Republic. BMC Microbiol. 2018;18(1):22.

39. Gomgnimbou MK, Abadia E, Zhang J, Refregier G, Panaiotov S, Bachiyska E, Sola C. $<<$ Spoligoriftyping $>>$ a DPO-based direct-hybridization assay for TB control on a multianalyte microbead-based hybridization system. J Clin Microbiol. 2012;50(10):3172-9.

40. Yasmin M, Gomgnimbou MK, Siddiqui RT, Refregier G, Sola C. Multi-drug resistant Mycobacterium tuberculosis complex genetic diversity and clues on recent transmission in Punjab, Pakistan. Infect Genet Evol. 2014;27C:6-14.

41. Dantas N, Suffys P, da Silva CW, Gomes HM, Neves de Almeida I, Jouca de Assis L, Gomgnimbou M, Refrégier G, Sola C, Spindola De Miranda S. Multidrug-resistant Mycobacterium tuberculosis genetic diversity in Minas Gerais state, Brazil. BMC Infectious Diseases. 2015;15:306.

42. Chaidir L, Sengstake S, de Beer J, Oktavian A, Krismawati H, Muhapril E, Kusumadewi I, Annisa J, Anthony R, van Soolingen D, et al. Predominance of modern Mycobacterium tuberculosis strains and active transmission of Beijing sublineage in Jayapura, Indonesia Papua. Infect Genet Evol. 2016;39:187-93.

43. Coll F, Phelan J, Hill-Cawthorne GA, Nair MB, Mallard K, Ali S, Abdallah AM, Alghamdi S, Alsomali M, Ahmed AO, et al. Genome-wide analysis of multiand extensively drug-resistant Mycobacterium tuberculosis. Nat Genet. 2018;50(2):307-16

44. Streicher EM, Bergval I, Dheda K, Bottger EC, Gey van Pittius NC, Bosman M, Coetzee G, Anthony RM, van Helden PD, Victor TC, et al. Mycobacterium tuberculosis population structure determines the outcome of genetics-based second-line drug resistance testing. Antimicrob Agents Chemother. 2012; 56(5):2420-7.

45. Ahmad S, Mokaddas E, Al-Mutairi N, Eldeen HS, Mohammadi S. Discordance across phenotypic and molecular methods for drug susceptibility testing of drug-resistant Mycobacterium tuberculosis isolates in a low TB incidence country. PLoS One. 2016;11(4):e0153563.

46. The CRyPTIC consortium Genomes Project, Allix-Beguec C, Arandjelovic I, Bi L, Beckert P, Bonnet M, Bradley P, Cabibbe AM, Cancino-Munoz I, et al. Prediction of susceptibility to first-line tuberculosis drugs by DNA sequencing. N Engl J Med. 2018;379(15):1403-15.

47. Makhado NA, Matabane E, Faccin M, Pincon C, Jouet A, Boutachkourt F, Goeminne L, Gaudin C, Maphalala G, Beckert P, et al. Outbreak of multidrugresistant tuberculosis in South Africa undetected by WHO-endorsed commercial tests: an observational study. Lancet Infect Dis. 2018;18(12): 1350-9.

48. Aung HL, Tun T, Nyunt WW, Fong Y, Crump JA, Thinn KK, Aung ST, Cook GM. Association between anti-tuberculosis drug resistance-conferring mutations and treatment outcomes in Myanmar. Infect Dis (Lond). 2018; 50(5):388-90.

49. Phelan J, O'Sullivan DM, Machado D, Ramos J, Whale AS, O'Grady J, Dheda K, Campino S, McNerney R, Viveiros M, et al. The variability and reproducibility of whole genome sequencing technology for detecting resistance to anti-tuberculous drugs. Genome medicine. 2016;8(1):132.

50. Borrell S, Teo Y, Giardina F, Streicher EM, Klopper M, Feldmann J, Muller B, Victor TC, Gagneux S. Epistasis between antibiotic resistance mutations drives the evolution of extensively drug-resistant tuberculosis. Evolution, medicine, and public health. 2013;2013(1):65-74

51. Anonymous: Tuberculosis Diagnostics Technology and Market Landscape. In. Geneva, UNITAID Secretariat Chemin de Blandonnet 10 - BIBC III - 8th Floor 1214 Vernier Switzerland: WHO; 2015: 1-96.

52. Anonymous: TB diagnostics market in select high-bruden countries : current market and future opportunities for novel diagnostics. In. Geneva, UNITAID Secretariat Chemin de Blandonnet 10 - BIBC III - 8th Floor 1214 Vernier Switzerland: WHO; 2015: 1-68.

53. Lecuit M, Eloit M. The potential of whole genome NGS for infectious disease diagnosis. Expert Rev Mol Diagn. 2015;15(12):1517-9.

54. Sidibe M. Democratising the global health agenda: why we need France. Lancet. 2016;6736(16):30060-5. 
55. Kehr J. 'Exotic no more': tuberculosis, public debt and global health in Berlin. Glob Public Health. 2018;13(3):369-82.

56. Sanchez-Padilla E, Merker M, Beckert P, Jochims F, Dlamini T, Kahn P, Bonnet M, Niemann S. Detection of drug-resistant tuberculosis by Xpert MTB/RIF in Swaziland. N Engl J Med. 2015;372(12):1181-2.

57. McNerney R, Cunningham J, Hepple P, Zumla A. New tuberculosis diagnostics and rollout. Int J Infect Dis. 2015;32:81-6.

58. Perez-Lago L, Izco S, Herranz M, Tudo G, Carcelen M, Comas I, Sierra O, Gonzalez-Martin J, Ruiz-Serrano MJ, Eyene J, et al. A novel strategy based on genomics and specific PCR reveals how a multidrug-resistant Mycobacterium tuberculosis strain became prevalent in Equatorial Guinea 15 years after its emergence. Clin Microbiol Infect. 2017;23(2):92-7.

59. Yasmin M, Refregier G, Siddiqui RT, labal R, Abbasi SA, Tahseen S. Reverse line probe assay for cheap detection of single nucleotide polymorphisms in Mycobacterium tuberculosis. Tuberculosis (Edinb). 2018:110:52-5.

60. Acosta F, Agapito J, Cabibbe AM, Caceres T, Sola C, Perez-Lago L, Abascal E, Herranz M, Meza E, Klotoe B, et al. Exportation of MDR TB to Europe from setting with actively transmitted persistent strains in Peru. Emerg Infect Dis. 2019;25(3):596-8

\section{Publisher's Note}

Springer Nature remains neutral with regard to jurisdictional claims in published maps and institutional affiliations.

Ready to submit your research? Choose BMC and benefit from:

- fast, convenient online submission

- thorough peer review by experienced researchers in your field

- rapid publication on acceptance

- support for research data, including large and complex data types

- gold Open Access which fosters wider collaboration and increased citations

- maximum visibility for your research: over $100 \mathrm{M}$ website views per year

At $\mathrm{BMC}$, research is always in progress.

Learn more biomedcentral.com/submissions 